\title{
Texture Features Extraction of Human Leather Ports Based on Histogram
}

\author{
Anita Sindar RM Sinaga \\ Departement of Informatics Engimeering, STMIK Pelita Nusantara Medan \\ Email: haito_ita@yahoo.com
}

\section{Article Info \\ Article history:}

Received Aug $10^{\text {th }}, 2018$

Revised Oct $10^{\text {th }}, 2018$

Accepted Oct $29^{\text {th }}, 2018$

\section{Keyword:}

Features

Histogram

Skin Pores

Textures

\begin{abstract}
Skin problems general are distinguished on healthy and unhealthy skin. Based on the pores, unhealthy skin: dry, moist or oily skin. Skin problems are identified from the image capture results. Skin image is processed using histogram method which aim to get skin type pattern. The study used 7 images classified by skin type, determined histogram, then extracted with features of average intensity, contrast, slope, energy, entropy and subtlety. Specified skin type reference as a skin test comparator. The histogram-based skin feature feature aims to determine the pattern of pore classification of human skin. The results of the 1,2, 3 leaf image testing were lean to normal skin (43\%), 4, 5, tends to dry skin (29\%), 6.7 tend to oily skin (29\%). Percentage of feature-based extraction of histogram in image processing reaches $90-95 \%$.
\end{abstract}

Copyright $@ 2018$ Puzzle Research of Data Technology

Corresponding Author:

Anita Sindar RM Sinaga

Department of Informatics Engineering

STMIK Pelita Nusantara

Jl. Iskandar Muda No. 1 Medan

Email: haito_ita@yahoo.com

\section{INTRODUCTION}

The skin is the largest organ in the body, an area of about $2 \mathrm{~m} 2$. The thickness of each body part varies $(0.5-5 \mathrm{~mm})$ and the average thickness is $1-2 \mathrm{~mm}$. Viewed from the texture, healthy skin feels softer and smoother, has moisture enough skin, not too oily, and not too dry so the skin looks fresh and supple. While unhealthy skin will feel uncomfortable when held, rough, feels stiff, and very sensitive if exposed to direct exposure to sunlight. Healthy skin, when pulled by the fingers will be more elastic and can re-fasten quickly, while unhealthy skin has a low skin elasticity [1]. The pattern obtained for each skin type. An image histogram is a graph depicting the deployment of pixel intensity values of a particular image or part of an image. Feature extraction is used to find certain patterns of different textural properties. Data in both static and dynamic forms consists of text, images, audio (voices, sounds, sounds, music), and videos.

The results of the data input process are computerized to obtain information from the input data process. The first texture used for segmentation is to separate one object with another object, second for texture classification. Pixel operation is an image processing operation that maps the relationship of each pixel that depends on the pixel itself. From a histogram can be known the frequency of emergence relative (relative) of the intensity of the image. The histogram can also show the brightness and contrast of an image. A histogram is a simple method of obtaining textures, it is helpful to observe the spread of color intensity, determine the boundaries of object separation from the background and give the percentage of color and texture composition [2]. The histogram can't be used to guess the shape of the object because it does not reflect the pixel color position arrangement. The term retrieval in image processing is used so that computers can identify objects like humans know the image. When finding the same texture from different images, it can be concluded that the image has the same features. In this research will be determined the value of intensity, brightness, size of asymmetry, distribution of pixel intensity of gray, maximum energy value, and roughness / intensity of image of dry, oily and normal skin type.

The histogram defines the characteristics of the image, for example, a narrowly distributed histogram indicated the low-contrast image. The features that can be extracted are mean, variance, skewness, kurtosis, energy, entropy. The other features which can be archived of the histogram are the maximum, 
minimum, median, and the range. The information of this histogram is used as features for texture segmentation [3]. Histogram equation is a popular feature compensation technique that has been well researched and practiced in image processing for normalizing digital visual features of images, such as brightness, gray-level scale, contrast, and so on. The image histogram shows the histogram of the pixel intensity value. The histogram denotes the number of pixels in an image grouped by different pixel intensity levels. In the 8-bit grayscale image, there are 256 different intensity level values then the histogram will be displayed graphically in the distribution of each 256-pixel level.

Skin segmentation is the basic of many important image processing systems. In this paper, we successfully improve the precision of previous GMM algorithm, which just considers the color feature as unique segmentation feature, caused a lot of skin color-like object detect as skin, that increase the FPR. We proposed a skin color segmentation system which combine color and textures feature as segmentation feature, eliminate non-skin part that detect as skin by previous method, finally we got the average detection rate of our method by $90.3 \%$ true positive with $20.5 \%$ false positive [4]. Major goal of image feature extraction is given an image, or a region within an image, generate the features that will subsequently be fed to a classifier in order to classify the image in one of the possible classes. The goal is to generate features that packing properties to identification skin type human leather ports based on histogram.

\section{RESEARCH METHOD}

The first phase of skin features extraction research is the second stage, perform preprocessing and feature extraction to know the average of intensity, contrast, skewness, energy, entropy and smoothness. The next step, comparing the extraction of the test image features with 3 reference imagery. An image can be defined as a two-dimensional function $\mathrm{f}(\mathrm{x}, \mathrm{y})$ where $\mathrm{x} \& \mathrm{y}$ is the field coordinate and the magnitude of $\mathrm{f}(\mathrm{x}$, $\mathrm{y})$ is the light intensity of the image at a point (x, y). The digital image is an image that has been through the process of sampling and quantizing.

A picture is continuous, by doing the sampling process \& quantizing, the image is changed to be discrete. The sampling process is the process of converting the image coordinates into a discrete nature, called pixels. The quantizing process is the process of assigning an intensity value to each pixel. The contrast image is defined by the dynamic range, defined as the ratio between the brightest and darkest parts of the pixel intensity. The histogram provides information for the contrast and the overall intensity of the distribution of an image. Suppose the input image $\mathrm{f}(\mathrm{x}, \mathrm{y})$ consists of a discrete gray level in the dynamic range $[0, \mathrm{~L}-1][5]$.

Image analysis considers the image for a specific application and it is useful in many applications such as computer vision, medical imaging, and pattern recognition. It extracts the useful information from the image. The image analysis involves image segmentation, image transformation, pattern classification, and feature extraction.

Building a vector of an image is done by merging rows of an image placed adjacent to the other rows in sequence. The texture is used as an image retrieval feature because some objects have certain patterns. The texture is the mutual relationship between the value of the intensity of repeated neighboring pixels in a large area rather than the distance of the relationship. Some texture applications, automatic inspection of the textile industry, car painting, carpet usage, medical image analysis, remote sensing analysis. In general, image classification is built based on color, texture and shape features which are used independently or in combinatorial.

From those features, color, feature becomes the most dominant and is broadly used for discriminating images. The objective of color indexing compares an image query to images in the database. The next stage is the detection of texton on the result of colour quantization and edge orientation quantization. Element size is used in this paper to increase texture discrimination, because the texture gradients contain only at texture boundaries [6]. The histogram is a graph that can describe the process of spreading pixel intensity values. In grayish-scale imagery, the number of gray levels (symbol L) starts from 0 to 255. For color images, a combined RGB component is applied. An image histogram is a diagram that describes the frequency of each intensity value that appears across the pixels. Grayscale imagery is an image that only uses color on a gray color level. Gray, the only color in RGB space with red, green, and blue components has the same intensity.

The gray-shaded image needs to express the intensity value for each pixel as a single value, whereas in the color image it needs three intensity values for each pixel. The histogram is the statistical probability distribution of each gray level in a digital image. The histogram provides contrast information and the overall intensity of the distribution of an image. Operation on the histogram improves brightness, stretches contrast, brightness and contrast combination, reverses the image, linear mapping, gray level cutting, and histogram exclusion [7]. Skin segmentation is the basis of many important image processing systems. The proposed a 
skin color segmentation system which combine color and textures feature as segmentation feature, eliminate non-skin part that detect as skin by previous method, finally got the average detection.

Generally the transformation of an image provides the frequency domain information of the data. The transform features of an image are extracted using zonal filtering. This is also called as feature mask, feature mask being a slit or an aperture. The high frequency components are commonly used for boundary and edge detection. Transform feature extraction is also important when the input data originates in the transform coordinate [8].

The color histogram can not only easily characterize the global and regional distribution of colors in an image, but also be invariant to rotation about the view axis. In color histograms, quantization is a process where number of bins is reduced by taking colors that are similar to each other and placing them in the same bin. The complexity of the algorithm also reduced by using these texture features. A feature known statically in a histogram is three images that contain different textures [9].

1. Intensity Average

The first feature that is calculated statically is the average intensity, calculated based on the equation:

$$
\mathrm{m}=\sum_{\mathrm{i}=0}^{\mathrm{L}-1} \mathrm{i} \cdot \mathrm{p}(\mathrm{i})
$$

\section{Brightness Average} calculation is:

Equation produces the average brightness of the object, this feature is standard deviation, the

$$
\sigma=\sqrt{\sum_{1=0}^{\mathrm{L}-1}(\mathrm{i}-\mathrm{m})^{2} \mathrm{p}(\mathrm{i})}
$$

$\sigma$ Called variance or a normalized second-order moment because p(i) is a function of giving an opportunity, giving a measure of contrast.

\section{Skewness}

The skewness feature is a measure of asymmetry to the mean intensity. Skewness is often called a normalized three-order moment. The negative value states that the brightness distribution is leaning to the left against the mean.

$$
\text { skewness }=\sum_{i=0}^{L-1}(i-m)^{3} p(i)
$$

Practice the value of skewness divided by $(\mathrm{L}-1)^{2}$ to be normalized.

\section{Descriptor energy}

The energy descriptor is a measure expressing the intensity distribution of pixels to gray, the equation:

$$
\text { energy }=\sum_{i=0}^{L-1}[p(i)]^{2}
$$

5. Entropy

A uniform image with a gray level of value will have a maximum energy value, which is equal to 1 . Uniformity or entropy ie the image with a slight gray level will have higher energy than that has a lot of gray levels.

$$
\text { entropy }=\sum_{i=0}^{L-1} p(i) \log _{2}(p(i))
$$

6. Smoothness

Smoothness is included to measure the smoothness level/roughness of image intensity, with the equation:

$$
\mathrm{R}=1-\frac{1}{1+\sigma^{2}}
$$


$\sigma$ is the standard deviation, the low R-value indicates the image has a rough intensity. Calculating fineness, the variance is normalized so that it is within the range [ 01 1] by dividing by $(\mathrm{L}-1)^{2}$. The histogram features considered in this paper are statistically based in that the histogram models the probability distribution of intensity levels in the image. These statistical features encode characteristics of the intensity level distribution for the image. A bright image will have a high mean and a dark image will have a low mean.

\section{RESULTS AND ANALYSIS}

Proposed method starts with the conversion of the input RGB image into a single component grayscale image to reduce the computation cost because the color image consists of Red, Green and Red components. Extract the information from the raw data that is most relevant for discrimination between the classes. Extract features with low within-class variability and high between class variability. Discard redundant information. First order statistics (information related to the gray level distribution) on histogram (texture) features. Second order statistics (information related to spatial/relative distribution of gray level), i.e. second order histogram, co-occurrence matrix. The grayscale image is converted into a histogram equalized image, to make the image's intensity levels equal to get a high contrast image. The image is expressed as a matrix, the size assumed to be $\mathrm{M}$ x $\mathrm{N}$ pixels, each pixel encoded 8 bits or 256 gray levels in Figure 1a, 1b.
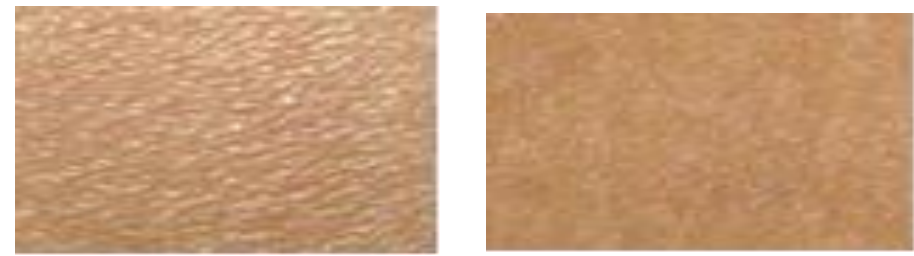

Figure 1 (a). Cropping Normal Skin 291x231 Pixels (b). Resize Normal Skin 300x300 Pixels

The histogram of an image refers to intensity values of pixels. The histogram shows the number of pixels in an image at each intensity value. The image in this study using the image of human skin pore amounted to 7 type file PNG not yet known skin type of each image and 3 images of reference. The image obtained from shoot camera phone is stored in the JPEG file. A good image has a histogram that fills the gray-level region in full with a uniform distribution of each pixel of intensity value in Figure 2 . The Imshist function calculates the number of pixels of an image for each color range (0-255). The Imhist function is designed to display an image histogram in grayscale format.

Reference imagery of Figure 2, 3 and 4, viewed from the top of the histogram, the dry skin texture image has an intense area of 150 - 200, the normal skin texture image has 150 intensities, the image of oily skin texture has an intensity. Image preprocessing is the process of resizing the image and then convert the image to grayscale. In plain view can be known skin type, by holding the skin, then taken 3 images as a reference image, Figure 2: normal skin, dry skin, Oily skin.
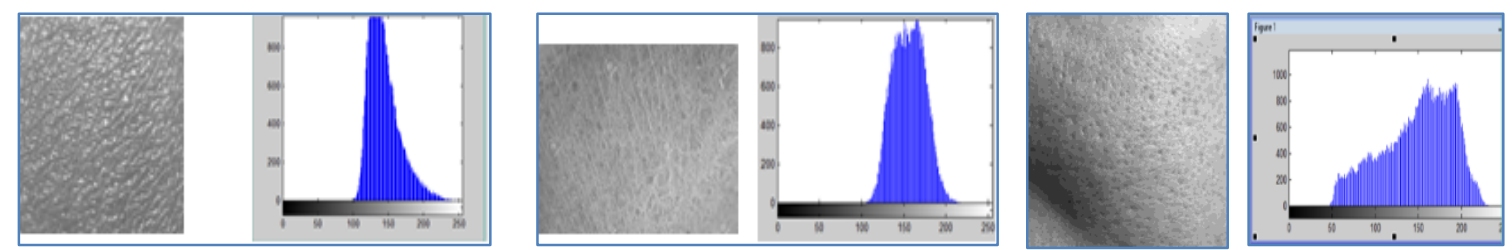

Figure 2. Normal Skin, Dry Skin, Oily Skin Texture and Imhist Result

Based on the repetition of patterns in the image, the texture is categorized into two forms, regular texture and irregular texture. Man-made textures tend to be orderly and natural textures tend to be irregular. In terms of the order, the pattern is easily recognized by the eye, to obtain the level of accuracy required method of recognition of texture. The peak of the histogram shows the average pixel intensity. The width of the peak shows the contrast ranges of the image. An image that is either overexposed or too dark (underexposed) has a narrow histogram.

Texture analysis is an essential issue in computer vision and image processing, such as in remote sensing, content based image retrieval, and so on. Texture analysis has steps:

1. Texture extraction: in the first step of texture analysis, some basic information on the image that is useful for other steps is calculated. Homogeneity or similarity between different regions of an image. 
2. Texture classification: an image is assigned to one of a set of predefined texture classes. Each class contains similar texture samples.

3. Texture segmentation: texture segmentation is done to partition a textured image into a set of homogeneous disjointed regions so each region contains a single texture based on texture properties.

4. Shape from texture: its methods provide information about shape from texture deformation features in an image.

The histogram tends to contain the color gray, high low intensity values can be seen from the comparison of the color gray with white color, the top of the histogram of the average intensity of 500 positions around 40-50.

Energy can be defined as the measure of the extent of pixel pair repetitions. It measures the uniformity of an image. When pixels are very similar, the energy value will be large. Entropy is the measure of randomness that is used to characterize the texture of the input image. Its value will be maximum when all the elements of the co-occurrence matrix are the same.

Taken testing data and stored in a database image results in the database later will be compared with the reference image so that the image obtained close to the skin classification on Figure 3.
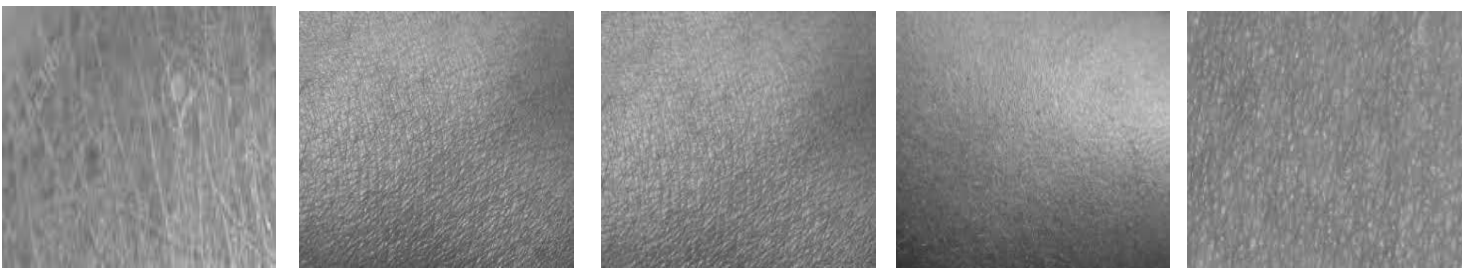

Figure 3. Cropped, Resize and Grayscale Testing Image Result

Energy can be defined as the measure of the extent of pixel pair repetitions. It measures the uniformity of an image. When pixels are very similar, the energy value will be large. Entropy is the measure of randomness that is used to characterize the texture of the input image. Its value will be maximum when all the elements of the co-occurrence matrix are the same. Extract feature testing image result on histogram on Table 1 and extract feature reference image result on histogram on Table 2.

Table 1. Extract Feature Testing Image Result on Histogram

\begin{tabular}{ccccccc}
\hline & $\begin{array}{c}\text { Intensity } \\
\text { Average }\end{array}$ & $\begin{array}{c}\text { Brightness } \\
\text { Average }\end{array}$ & Skewness & $\begin{array}{c}\text { Deskriptor } \\
\text { Energy }\end{array}$ & Entropy & Smoothness \\
\hline 1 & 0.64400 & 0.0016 & 0.00808 & $3.0581 \mathrm{E}+13$ & 6.5151 & 0.00256 \\
2 & 0.00503 & 10.4881 & 0.00495 & $3.0581 \mathrm{E}+13$ & 6.4493 & 251.006 \\
3 & 0.00503 & 0.0706 & 0.00503 & 0.000025301 & 6.4502 & 0.0055 \\
4 & 0.00866 & 0.0815 & 0.00659 & 0.000043428 & 6.9627 & 0.0066 \\
5 & 0.00493 & 15.8900 & 0.00485 & 0.000024305 & 5.4225 & 252.492 \\
6 & 0.00018 & 88.7694 & 0.00189 & $3.5721 \mathrm{E}-12$ & 6.2666 & $7.88 \mathrm{E}+03$ \\
7 & 0.00510 & 0.001148 & $6.74 \mathrm{E}-10$ & 0.00002601 & 6.1897 & $1.32 \mathrm{E}-10$ \\
\hline
\end{tabular}

Table 2. Extract Feature Reference Image Result on Histogram

\begin{tabular}{cccccrr}
\hline Skin & Intensity & Brightness & Reference Image \\
Average & Average & Skewness & $\begin{array}{c}\text { Deskriptor } \\
\text { Energy }\end{array}$ & Entropy & Smoothness \\
\hline Normal & 0.00478 & 0.0691 & 0.00478 & $2.285 \mathrm{E}-05$ & 6.4043 & 0.0048 \\
Dry & 0.00507 & 0.0709 & 0.005 & 6.2531 & 6.2531 & 0.0705 \\
Oily & 0.00283 & 0.8597 & 0.722 & 0.7391 & 7.2651 & 0.7391 \\
\hline
\end{tabular}

High contrast regions have high variance, and low contrast images have low variance. The skew is positive when the tail of the histogram spreads out to the right (positive side), and is negative when the tail of the histogram spreads out to the left (negative side). High energy means that the number of different intensity levels in the region is low, the distribution is concentrated over only a small number of different intensity levels.

These quantities can be used to characterize the image texture. Entropy is a measure of the number of bits required to encode the region data. Entropy increases as the pixel values in the image are distributed among a larger number of intensity levels. Complex regions have higher entropy and entropy tends to vary inversely with energy. Entropy is a measure of variability. It zero for a homogenous image (all the pixels have a similar values). Where $\sigma$ is the standard deviation. Kurtosis measures the peakedness of the distribution. The percentage of image imagery feature approach to reference image result on Table 3. 
Table 3. Percentage of Image Imagery Feature Approach to Reference Image

\begin{tabular}{ccc}
\hline & Image Normal Skin & \\
\hline intensity average & image 1, 3 & $96 \%$ \\
brightness average & image 2, 3 & $95 \%$ \\
skewness & image 1 & $95 \%$ \\
entropy & image 2 & $96 \%$ \\
energy & image 3 & $94 \%$ \\
smoothness & image 3 & $96 \%$ \\
\hline intensity average & & $95 \%$ \\
brightness average & image 2, 3, 7 & $89 \%$ \\
skewness & image 6, 7 & $99 \%$ \\
entropy & image 6 & $97 \%$ \\
energy & image 6, 7 & $87 \%$ \\
smoothness & image 7 & - \\
\hline & - & $96 \%$ \\
intensity average & & $95 \%$ \\
brightness average & image 5 & $95 \%$ \\
skewness & image 4 & $95 \%$ \\
entropy & image 5 & $94 \%$ \\
energy & image 4 & $92 \%$ \\
\hline smoothness & image 5 & \\
\hline
\end{tabular}

The results of the extract-based feature of image-histogram-based testing were compared with reference imagery, 1, 2, 3 leanings of normal skin (43\%), 4, 5 inclined oily skin (29\%), 6, 7 leanings of dry skin $(29 \%)$.

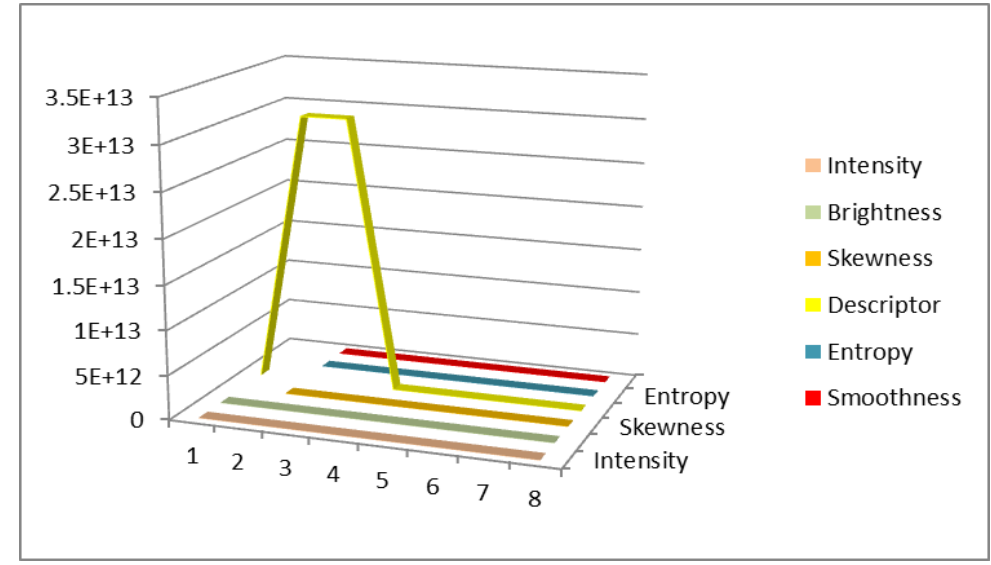

Figure 4. The Comparison Result of Texture Features Extraction of Human Leather Ports Based on Histogram

The comparison results of each image testing extraction is on Figure 6. The dominant value that is entropy, skewness and intensity. The most dominant value of image testing is descriptor energy. For image testing 2 and 3 has the highest descriptor energy. Histogram-based feature extraction calculations tend to compare the gray color content of the image. The high-intensity averages are supported with high gray values, affecting the intensity distribution of pixels to gray (descriptor energy). The highest intensity level is in image 6: 0.00189 (tend to dry skin); image brightness highest: 0.011489 (tend to dry skin); entropy highest: 6.9627 (tends to oily skin).

\section{CONCLUSION}

Histogram-based feature extraction calculations tend to compare the gray color content of the image. The high-intensity averages are supported with high gray values, affecting the intensity distribution of pixels to gray (descriptor energy). The highest intensity level is in image 6: 0.00189 (tend to dry skin); image brightness highest: 0.011489 (tend to dry skin); entropy highest: 6.9627 (tends to oily skin). Dry skin has not smoothness value. Normal skin has an average value of intensity, brightness, skewness and smoothness are balanced. Based on the research result, the percentage of feature extraction based on the histogram in extract future approach reach $90-95 \%$. 


\section{ACKNOWLEDGEMENT}

The authors would like to thank all idea for writing and researching. For Library STMIK Penusa Medan. Very big thank.

\section{REFERENCES}

[1] Salamah SPE, Budiarti TA, "Formulation of Skin Lotion with Addition of Carrageenan and Natural Antioxidant from Rizophora Mucronata Lamk," Jurnal Akuatika, Vol. 5 No. 1, pp. 55 - 62, 2014.

[2] Verma R, Rajput V, “A Review on Histogram Value and Texture Descriptor Analysis for Image Retrieval”, International Research Journal of Engineering and Technology (IRJET), Vol. 03 No. 04, pp.2132-2135, 2016.

[3] Zayed N, Elnemr HA, "Statistical Analysis of Haralick Texture Features to Discriminate Lung Abnormalities", Computer \& Systems Department, Electronics Research Institute, Cairo 12611, 2015.

[4] Kiadtikornthaweeyot W, Tatnall ARL, "Region Of Interest Detection Based On Histogram Segmentation For Satellite Image", The International Archives of the Photogrammetry, Remote Sensing and Spatial Information Sciences, Volume XLI-B7, XXIII ISPRS Congress, Prague, Czech Republic, pp. 12 - 19, 2016.

[5] Minarno AE, Munarko Y, Kurniawardhani A, Bimantoro F, "Classification of Texture Using Multi Texton Histogram and Probabilistic Neural Network”, IOP Conf. Series: Materials Science and Engineering, 2016.

[6] Mali SN, Tejaswini ML, “Color Histogram Features for Image Retrieval Systems", International Journal of Innovative Research in Science, Engineering and Technology, Vol. 3, No. 4, pp. 1094-10946, 2014.

[7] Zhang X, Cui J, Wang W, Lin C, "A Study for Texture Feature Extraction of High-Resolution Satellite Images Based on Direction Measure and Gray Level Co-Occurrence Matrix Fusion Algorithm”, Sensors, pp. 2 - 15, 2017.

[8] Suresh MB, Naik BM, "Content Based Image Retrieval Using Texture Structure Histogram and Texture Features", International Journal of Computational Intelligence Research, Vol. 13, No. 9, pp. 2237-2245, 2017.

[9] Bahadure NB, Ray AK, Thethi HP, "Image Analysis for MRI Based Brain Tumor Detection and Feature Extraction Using Biologically Inspired BWT and SVM”, International Journal of Biomedical Imaging, pp. 1-12, 2017.

\section{BIBLIOGRAPHY OF AUTHORS}

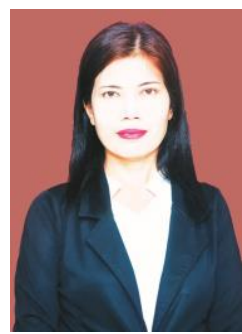

Author is former Director of NNI Poliprofesi. Former Director of Polytechnic Gihon. Currently as a lecturer in Informatics Engineering STMIK Pelita Nusantara Medan. Join in LPPM STMIK Penusa Medan 